\title{
MRI and Pregnancy
}

\author{
Pasquini $\mathrm{L}^{1 *}$ and Di Stasio $\mathrm{M}^{2}$ \\ ${ }^{1}$ Neuroradiology Unit, NESMOS Department, La Sapienza University, Rome \\ ${ }^{2}$ Radiology Units, Department of Experimental and Clinical Biomedical Sciences, University of Florence - AOU Florence
}

Received: March 18, 2018; Published: March 29, 2018

*Corresponding author: Pasquini L, Neuro radiology Unit, Sant'Andrea Hospital, Via di Grottarossa 1035, 00189, Rome, Tel: 06.3377.5225;

Email: lucapasquini3@gmail.com

Abbreviations: MRI: Magnetic Resonance Imaging; GBCAs: Gadolinium-Based Contrast Agents; FAD: Food and Drug Administration; EMA: European Medicines Agency

\section{Introduction}

Magnetic Resonance Imaging (MRI) is indicated during pregnancy for both foetal pathology (CNS abnormalities, neck and oropharynx masses, diaphragmatic hernia, placental pathology, abdominal masses or intestinal pathology without sufficient characterization at ultrasound, suspected foetal infection) and maternal pathology (acute abdomen, appendicitis) [1,2]. The developing human foetus is considered particularly vulnerable to external insults, so that the possible consequences of MRI during pregnancy have been widely investigated to isolate possible side effects and identify the best safety parameters. The anatomy of the human foetus changes every week of gestation, affecting the interactions with the external environment. The development of the internal organs which are deputed to distribute and excrete chemicals (e.g.) strongly affects the pharmacokinetics of contrast agents. The main foetal risks during MR examinations include GBCArelated effects (nephrogenic system fibrosis - NSF -, teratogenicity, brain deposit) and physical effects (body temperature increase, acoustic injuries related to the scanner noise).

\section{GBCAS-Related Risk Factors}

Gadolinium-based contrast agents (GBCAs) administration is currently discouraged in ordinary foetal examinations. The ESUR 9.0 (2014) guidelines restrict the prenatal use of GBCAs only to those cases with a clear clinical indication, recommending the administration of the lowest possible dose and the use of a stable contrast medium (low- or intermediate- risk class, macrocyclic contrast agents). In the case of poor maternal renal function, GBCAs are contraindicated [3]. The US Food and Drug Administration (FDA) [4] consider the GBCAs as part of the class C drugs (used only if their benefits outweigh the risks for the foetus). More recently, the European Medicines Agency (EMA) concluded its review on
GBCAs, confirming its recommendations to restrict the use of some linear Gadolinium-based agents in MRI body scans and to suspend the authorisation of others, particularly the intravenous linear products. Moreover, the same agency discourages the use of any contrast agent during pregnancy [5].

A recent study from Ray et al. [6] investigates the effects of MR imaging and Gadolinium (Gd) administration during pregnancy, with special regards to the first trimester, studying possible long-term side effects after 4 years of follow-up. The Authors retrospectively analysed 1737 women who underwent an MR examination during the first trimester of their pregnancy and 397 women who received GBCAs anytime during their pregnancy; both groups were compared to a large population of MR-unexposed subjects $(1,418,451)$. The study showed similar incidence of abortion (starting from the 21st week), congenital abnormalities, tumors and hearing impairment in controls and patients who performed an MR examination during the first trimester of pregnancy. Minimal visual deficits were identified only in a group of patients exposed between the 5 th and the 10th week (adjusted HR 2.28; 95\% CI, 1.09-4.77). In the GBCAsexposed group a positive correlation between GBCA administration during pregnancy, increased stillbirth and neonatal death was identified. Moreover, GBCAs exposure during the first trimester was associated with higher risk of rheumatological, inflammatory, or infiltrative dermatologic conditions (adjusted hazard ratio 1.41; 95\% confidence interval 1.11-1.79).

Overall, the study supports the limitation of foetal MRI during the first trimester of pregnancy to those patients showing clear clinical indications, not eligible for ultrasound investigation. The pharmacokinetic of GBCAs during foetal life is characterized by the "recirculation phenomena": contrast mediums access the foetal 
bloodstream trough the blood-placental barrier and are excreted trough the foetal kidneys into the amniotic fluid. For the fact that the foetus continuously ingests small amounts of amniotic fluid, GBCAs will recirculate until a balance between their haematic and amniotic concentration is reached. This on-going recirculation may facilitate the dissociation of $\mathrm{Gd}$ contrasts, resulting in release of free Gd and formation of toxic Gd salts [7]. Interestingly, a recent study on non-human primates reported lower concentrations of gadoteridol in the foetal-placental circulation rather than in the maternal bloodstream, with subsequent decrease 45 hours after the administration and negligible Gd concentrations in liver and bones [8].

Such results may partially mitigate the concerns about Gd pharmacokinetic in humans. However, Ray at al. found a significant correlation between maternal GBCA administration during the first trimester of pregnancy and late rheumatologic, inflammatory/ infiltrative dermatologic conditions in children [6], highlighting the special need of further investigation about the consequences of the administration of Gd contrasts during this vulnerable period, including long-term follow-ups and more sophisticate animal models. Particularly, the recent evidence of brain GBCA deposit in both the adult and the pediatric population with regards to different classes of contrast agents raises the legitimate suspicion of similar dynamics with foetal contrast-enhanced MRI [7, 9-11].

\section{Physical Effects}

The development of foetal abnormalities in response to temperature modifications has been demonstrated in animal models, showing teratogenic effects for about $2^{\circ} \mathrm{C}$ of increase in maternal temperature for more than 30 minutes or $4^{\circ} \mathrm{C}$ for more than 15 minutes [12]. More recently, a study by Cannie et al. investigated the correlation between the specific sequence acquired during the MR examination and the increment in body temperature of pig foetuses; particularly, low SAR (Specific Absorption Rate) sequences were associated with $<1^{\circ} \mathrm{C}$ variation of body temperature after 30 minutes of scanning [13]. Taking in account those evidences, a limitation to the foetal MR acquisition time up to 30 minutes on $1.5 \mathrm{~T}$ scanners seems reasonable, due possible heatingrelated risks for the foetus. Such recommendations currently lack for 3T foetal MRI, although no side effects have been reported so far [14]. MRI generates loud noise mainly due to the movement of the gradients. The acoustic waves interact with the foetus, challenging the developing inner ear with mechanical insults that could theoretically lead to hearing abnormalities after birth. However, such a putative relationship between foetal MR exposure and neonatal hearing injury has never been demonstrated in humans [15], and the study from Ray et al. may suggest the absence of such injuries even after MR-exposure during the first trimester [6].

\section{Conclusion}

Currently, there is no clear contraindication to MRI in any trimester of pregnancy; however the literature strongly recommends a careful case by case evaluation of patients, with accurate risk- benefit assessment. [16] Some evidences may suggest to limit the foetal MR acquisition time to 30 minutes on $1.5 \mathrm{~T}$ scanners, due to possible heating-related risks for the foetus $[12,13]$. According to FDA, ESUR and EMA recommendations on contrast administration, the use of GBCAs during pregnancy is not contraindicated and should not be avoided in case of clear clinical indications. However, the administration should be carefully evaluated in terms of riskbenefit ratio and the choice of the most stable GBCAs (macrocyclic molecules) is strongly recommended [3-5].

\section{References}

1. Bahado-Singh R, Goncalves L (2013) Techniques, terminology, and indications for MRI in pregnancy. Seminars in Perinatology 37(5): 334339.

2. Millischer A, Salomon L, Porcher R, Brasseur-Daudruy M, Gourdier A, et al. (2016) Magnetic resonance imaging for abnormally invasive placenta: the added value of intravenous gadolinium injection. BJOG: An International Journal of Obstetrics \& Gynaecology 124(1): 88-95.

3. (2016) ESUR Guidelines.

4. (2016) US Food and Drug Administration Home Page.

5. (2017) European Medicines Agency.

6. Ray J, Vermeulen M, Bharatha A, Montanera W, Park A (2016) Association Between MRI Exposure During Pregnancy and Fetal and Childhood Outcomes. JAMA 316(9): 952-961.

7. Pasquini L, Napolitano A, Visconti E, Longo D, Romano A, et al. (2018) Gadolinium-Based Contrast Agent-Related Toxicities. CNS Drugs.

8. Oh K, Roberts V, Schabel M, Grove K, Woods M, et al. (2015) Gadolinium Chelate Contrast Material in Pregnancy: Fetal Biodistribution in the Nonhuman Primate. Radiology 276(1): 110-118.

9. Pasquini L, Rossi Espagnet M, Napolitano A, Longo D, Bertaina A, et al. (2018) Dentate nucleus T1 hyperintensity: is it always gadolinium all that glitters? La radiologia medica.

10. Rossi Espagnet M, Bernardi B, Pasquini L, Figà-Talamanca L, Tomà $P$, et al. (2017) Signal intensity at unenhanced T1-weighted magnetic resonance in the globus pallidus and dentate nucleus after serial administrations of a macrocyclic gadolinium-based contrast agent in children. Pediatric Radiology 47(10): 1345-1352.

11. Ryu Y, Choi Y, Cheon J, Lee W, Park S, et al. (2018) Pediatric Brain. Investigative Radiology 53(4): 246-255.

12.Ziskin M, Morrissey J (2011) Thermal thresholds for teratogenicity, reproduction, and development. International Journal of Hyperthermia 27(4): 374-387.

13. Cannie M, De Keyzer F, Van Laere S, Leus A, de Mey J, et al. (2016) Potential Heating Effect in the Gravid Uterus by Using 3-T MR Imaging Protocols: Experimental Study in Miniature Pigs. Radiology 279(3): 754761.

14. Victoria T, Johnson A, Edgar J, Zarnow D, Vossough A, et al. (2016) Comparison Between 1.5-T and 3-T MRI for Fetal Imaging: Is There an Advantage to Imaging With a Higher Field Strength? American Journal of Roentgenology 206(1): 195-201.

15. Strizek B, Jani J, Mucyo E, De Keyzer F, Pauwels I, et al. (2015) Safety of MR Imaging at $1.5 \mathrm{~T}$ in Fetuses: A Retrospective Case-Control Study of Birth Weights and the Effects of Acoustic Noise. Radiology 275(2): 530537.

16. Kanal E, Barkovich A, Bell C, Borgstede J, Bradley W, et al. (2007) ACR Guidance Document for Safe MR Practices: 2007. American Journal of Roentgenology 188(6): 1447-1474. 
(C) (i) This work is licensed under Creative

Submission Link: https://biomedres.us/submit-manuscript.php

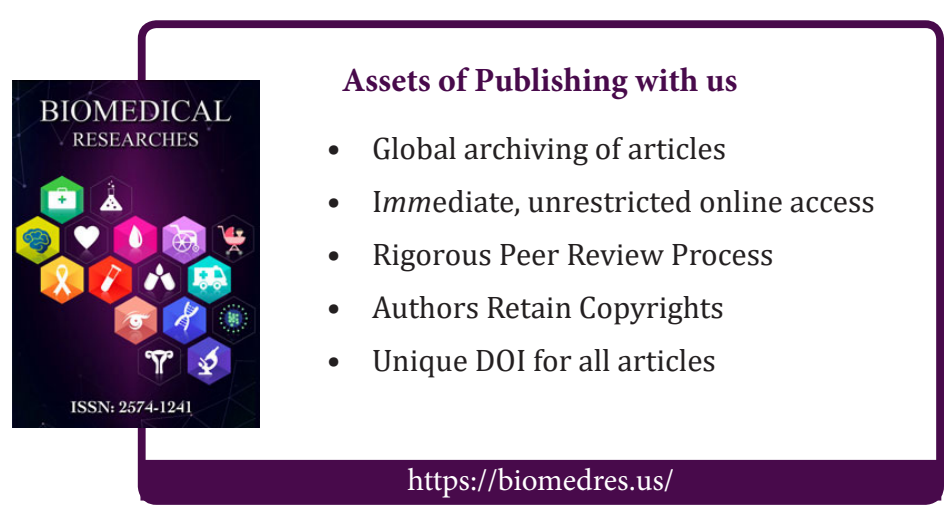

